\title{
Fluorescent PIV using Atomized Liquid Particles
}

\author{
Adit S. Acharya ${ }^{1 *}$, K. Todd Lowe', Wing F. Ng ${ }^{1}$ \\ ${ }^{1}$ Virginia Tech, Advanced Propulsion and Power Laboratory, Blacksburg, VA, USA \\ * aacharya@vt.edu
}

\begin{abstract}
It is shown that aerosolized fluorescent particles generated using a Venturi-type atomizer, from a solution of fluorescent Kiton Red 620 dye in a water/glycol fluid, provide effective flow seeding for fluorescent PIV. The atomized liquid particles were found to be of acceptable size for PIV purposes, with $92 \%$ of detected particles by number concentration measuring $<1 \mu \mathrm{m}$ in diameter. A PIV application was conducted in a wind tunnel (freestream velocity $U_{\infty}=27 \mathrm{~m} / \mathrm{s}$ ), using the particles for measurement of the boundary layer flow approaching a forward-facing step (approach boundary layer momentum thickness Reynolds number of $R e_{\theta}=5930$ ), to identify potential benefits in near-wall regions normally affected by unwanted laser reflections from tunnel surfaces. Particles were generated from solutions with dye molar concentrations of $2.5 \times 10^{-3}$ and $1.0 \times 10^{-2} \mathrm{~mol} / \mathrm{L}$, and PIV images were obtained for both elastic Mie scattering and filtered, Stokes-shifted fluorescent light. Raw images indicate that the fluorescence yield of the $1.0 \times 10^{-2} \mathrm{~mol} / \mathrm{L}$ solution provides PIV images with high contrast, even in the near-surface regions where Mie scattering images are highly affected by surface reflections. Boundary layer profiles are processed in the adverse pressure gradient region leading up to the forward-facing step, where the fluorescent PIV performed comparably to the most optimized Mie scattering PIV; both obtained data as near to the wall as $30 \mu \mathrm{m}$, or 2 viscous wall units in our flow of interest. These results indicate that the new seeding method holds excellent promise for near-surface measurement applications with more complicated three-dimensional geometries, where it is impossible to arrange PIV cameras to reject surface-scattered light.
\end{abstract}

\section{Introduction}

Particle image velocimetry (PIV), which involves the optical measurement of light-scattering tracer particles, has become a ubiquitous flow diagnostic technique over the past few decades. In PIV, a fluid flow of interest is seeded with small particles which have negligible inertia compared to drag forces (i.e., a low Stokes number) and thus effectively follow streamlines [Raffel et al. (2018)]. The particles are illuminated with laser light, which they scatter elastically at the wavelength of the incident laser (i.e., Mie scattering, in the case of spherical particles). Their motion is recorded with a quick succession of photographs, after which their velocities are obtained by image cross-correlation.

A frequent challenge for PIV experimentalists is the occurrence of surface laser reflection, or "flare", in flows around models [Paterna et al. (2013)], flows involving free surfaces [Pedocchi et al. (2008)], and studies of near-wall regions in general [Cadel et al. (2016)]. Flare is detrimental to PIV correlations because it decreases the image contrast and signal-to-noise ratio (SNR) in the vicinity of surfaces. Light that is scattered by surfaces can be far more intense than particle light scattering, potentially leading to saturation of sensor pixels and the loss of flow vectors [Chennaoui et al. (2008)]. Paterna et al. (2013) also note the possibility of camera sensor damage from intense flare, although with modern CMOS cameras this is less of a concern.

Attempts to reduce laser flare often involve modifications to surfaces themselves, such as a coat of black paint [Wernet (2000)] or clear surfaces that match the fluid's index of refraction [Uzol et al. 
(2002)]. The comparative study of materials and surface treatments by Paterna et al. (2013) concluded that a coating of fluorescent paint was the most effective method of reducing flare in air-based experiments, given that no material can match the refractive index of air. The fluorescent paint technique relies on laser-induced fluorescence (LIF), the emission of light that is red-shifted relative to any absorbed laser light. This alteration of wavelength is also known as the Stokes shift (as opposed to much weaker, blue-shifted fluorescence, termed anti-Stokes shift). Surfaces covered with fluorescent paint emit Stokesshifted light that can be blocked with a band-pass filter to limit their impact on PIV measurements; Miescattered light at the laser wavelength from tracer particles is still allowed through.

Alternatively, laser flare reduction may be accomplished with the use of fluorescent tracer particles; their Stokes-shifted light may be collected through a long-pass filter that blocks surface reflections at the laser's wavelength. Fluorescent PIV has been used in water-based experiments [Poussou and Plesniak (2006)], but is more challenging for gas flows, which require smaller particles and careful consideration of particle inhalation hazards [Maisto et al. (2013)]. For example, the study by Chennaoui et al. (2008) successfully demonstrated flare reduction with fluorescent PIV in air, but made partial use of hazardous materials that warranted protective measures.

A more recent study by Petrosky et al. (2015) demonstrated PIV with fluorescent dye-doped particles, with the goal of avoiding hazardous substances; Kiton Red 620 (KR620) dye was selected for its low toxicity. The KR620 dye was used to dope Polystyrene Latex (PSL) microspheres, which are commonly used as PIV tracer particles [Raffel et al. (2018)]. The ensuing experiments successfully allowed for accurate PIV measurements very near the surface of a flat plate, where conventional PIV techniques were shown to be severely hampered by flare. This result was notable for its combined use of safe materials and tracer particles small enough to be suitable for gas flows; as noted by Petrosky et al., the intensity of fluorescent light from particles is typically two to three orders of magnitude lower than Mie scattering intensity, and this difference is exacerbated for small particles.

Notably, all examples of fluorescent PIV to this point have involved the use of solid tracer particles. While effective, solid particles bring about a variety of undesirable challenges. The generation of dye-doped particles can be cumbersome, involving an emulsion polymerization process [Wohl et al. (2015)] or the grounding and dispersal of solidified resin [Pedocchi et al. (2008)]. These processes can be lengthy and expensive, requiring special facilities and materials. While researchers may order dye-doped particles for delivery, doing so constrains an experiment to a limited set of dye concentrations, which can have an impact on fluorescent PIV results. In the current study, the authors expand on the work of Petrosky et al. (2015), exploring a novel method of liquid fluorescent tracer particle generation and injection that is relatively simple and inexpensive when compared to the aforementioned solid particle techniques. The particles are first shown to be of acceptable size for PIV purposes. Then, they are used in a PIV experiment in the boundary layer of a wind tunnel approaching a forward-facing step. Both fluorescent and Mie PIV are conducted and comparisons are made between their wall boundary layer profiles and skin friction coefficient results, showing the ability of the fluorescent PIV to perform comparably to the most optimized Mie-PIV case.

\section{Experimental Methods}

Liquid fluorescent tracer particles were generated with a method the authors recently developed for the seeding of high-pressure nozzles [Acharya et al. (2021)]. This method, depicted conceptually in Figure 1, involved two main components: the nozzle/preceding tubing, and a separate liquid reservoir.

The reservoir was connected to the throat of a Venturi contraction upstream of the nozzle. When both the nozzle and reservoir were supplied with the same total pressure, the static pressure drop at the Venturi throat created suction, drawing the reservoir's liquid into the nozzle tubing. Once in the tubing, strong shear forces atomized the liquid into fine particles. Note in Figure 1 the inclusion of a needle valve between the liquid reservoir and Venturi throat; this was added after the initial study to aid with mass flow rate control. 


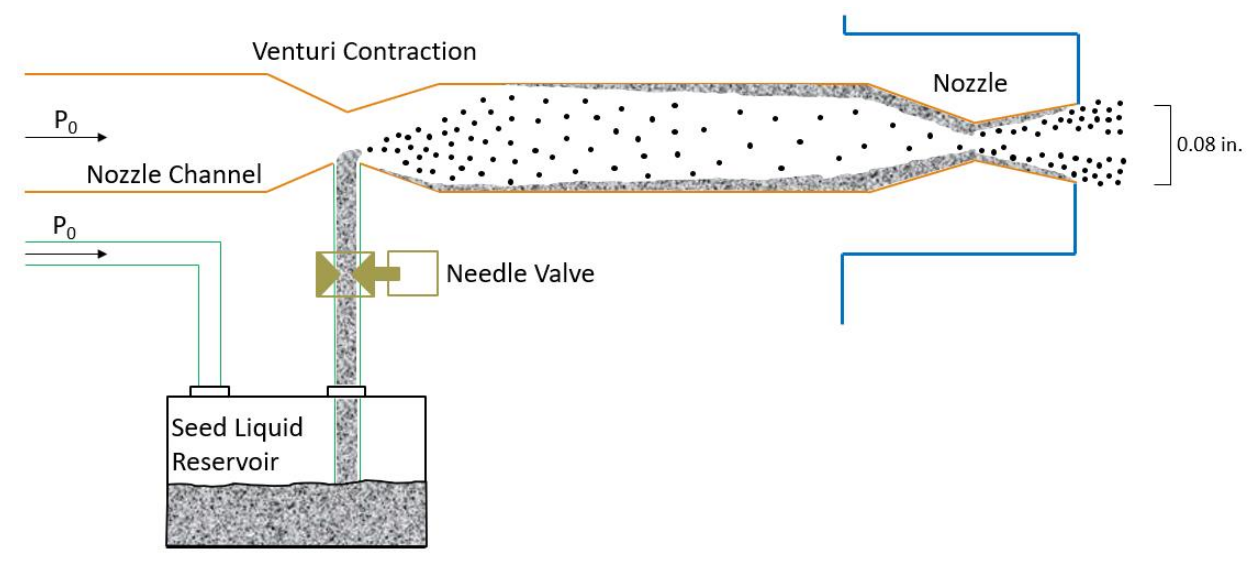

Figure 1: Schematic of Venturi seeding method for a high-pressure nozzle [Acharya et al. (2021)]

While the original intent of the device was for laser-based velocimetry and visualization of the nozzle plume itself, the authors saw potential in using the dense spray for PIV on external areas of interest, or even for seeding in wind tunnels. Additionally, it was thought that a solution of fluorescent dye in a suitable liquid would be atomized in the same way as depicted in Figure 1, creating fluorescent tracer particles for PIV. Kiton Red 620 dye was chosen for its safety and previous success in the study by Petrosky et al. (2015). The dye was dissolved in a non-toxic, proprietary water/glycol solution (i-fog Fluid, Martin Lighting), used for long-lasting fog generation. Two separate dye concentrations were used: $1.0 \times 10^{-2} \mathrm{~mol} / \mathrm{L}$ and $2.5 \times 10^{-3} \mathrm{~mol} / \mathrm{L}$.

Experiments aimed to assess the suitability of the generated particles for fluorescent PIV and to demonstrate the potential advantages of such a method for near-wall measurements. First, a TSI 3321 Aerodynamic Particle Sizer (APS) was used to extract and analyze particles from the nozzle's plume. This device determines aerodynamic size distributions of ingested particles over a range of 0.5 to $20 \mu \mathrm{m}$ in diameter. The Venturi device was supplied with $4.34 \mathrm{MPa}$ (630 psia) from a compressed nitrogen tank, with its reservoir filled with i-fog fluid and its needle valve $92 \%$ closed. This generated a spray that was directed at the inlet of the TSI 3321 APS for 30 seconds.

The sizing test was followed by a PIV experiment in the Small Boundary Layer (SBL) Wind Tunnel at Virginia Tech. The SBL tunnel is an open-circuit wind tunnel that features a blower capable of delivering $62.30 \mathrm{~m}^{3} / \mathrm{min}$ of air, a series of honeycombs and screens to eliminate large-scale turbulence, and a $24.13 \mathrm{~cm}$ x $11.03 \mathrm{~cm}$ x $199.39 \mathrm{~cm}$ acrylic/float glass optical test section with roughness introduced at the test section inlet for boundary layer tripping and thickening. The tunnel was operated such that the freestream flow velocity was nominally $27 \mathrm{~m} / \mathrm{s}$ in the test section. Relevant flow parameters were obtained with the PIV experiment (details below) and a Pitot-static probe; to fill in the outer region missed by the highly magnified PIV setup, PIV data were fit to a profile of $\frac{U}{U_{\infty}}=\left(\frac{y}{\delta}\right)^{\frac{1}{7}}$, where $U$ is velocity, $U_{\infty}$ is the velocity of the freestream, $y$ is the height above the wall, and $\delta$ is the boundary layer thickness. This allowed for determination of the thickness and approximate velocities for the full boundary layer. Flow parameters are shown in Table 1 , where $\mathrm{u}_{\tau}$ is friction velocity, WU is the wall unit height, $\delta *$ is displacement thickness, $\Theta$ is momentum thickness, $\operatorname{Re}_{\Theta}$ is momentum Reynolds number, and $\mathrm{H}$ is shape factor.

\begin{tabular}{c|cccccccc}
\multicolumn{8}{c}{ Table 1: SBL wind tunnel flow parameters } \\
Parameter & $\mathbf{U}_{\infty}$ & $\mathbf{u}_{\boldsymbol{\tau}}$ & $\mathbf{W U}$ & $\boldsymbol{\delta}$ & $\boldsymbol{\delta} *$ & $\boldsymbol{\Theta}$ & $\boldsymbol{R e}_{\boldsymbol{\Theta}}$ & $\mathbf{H}$ \\
\hline Value & 27 & 0.9880 & 15 & 39 & 4.29 & 3.25 & 5930 & 1.32 \\
Units & $\mathrm{m} / \mathrm{s}$ & $\mathrm{m} / \mathrm{s}$ & $\mu \mathrm{m}$ & $\mathrm{mm}$ & $\mathrm{mm}$ & $\mathrm{mm}$ & - & -
\end{tabular}


The PIV experimental setup is depicted in Figure 2. The Venturi seeding mechanism was placed such that its spray was directed into the suction inlet of the tunnel. From there, seeded flow passed through the blower, plenum, and contractions into the optical test section. There, a flat aluminum plate of height $4.78 \mathrm{~mm}$ was secured to the floor to create a forward-facing step flow configuration to challenge the measurement system. A two-dimensional/two-component PIV arrangement was used, in the common wall-grazing view imaging configuration optimized for boundary layer measurement [Willert (2015)]. An EverGreen dual-pulsed $532 \mathrm{~nm}$ laser was fitted with sheet-forming optics to produce a laser sheet aligned with the streamwise/normal-to-wall plane. A LaVision Imager sCMOS camera ( $2560 \times 2160$ pixels), fitted with a $100 \mathrm{~mm}$ focal length lens, was used to acquire images from a $32 \mathrm{~mm}$ x $32 \mathrm{~mm}$ region in the wall boundary layer, capturing the leading edge of the forward-facing step and a 27-mm-long section of the tunnel upstream of it. For fluorescent PIV measurements, an Omega Optical $560 \mathrm{~nm}$ long pass filter (model 560HLP) was attached to the camera lens to reject $532 \mathrm{~nm}$ laser light. It was expected, as per Petrosky et al. (2015), that KR620 dye would emit Stokes-shifted light mostly above $560 \mathrm{~nm}$. First, a set of 1000 Mie images was taken with the lens aperture set at f/2.8, the widest possible aperture setting. Another set of 1000 Mie images was taken with the aperture set to $f / 22$, reducing flare and particle intensities alike. Finally, the aperture was set to $\mathrm{f} / 2.8$ and the high-pass filter was attached to the lens. Two sets of 1000 fluorescent images were taken; one for a dye concentration of $1.0 \times 10^{-2}$ moles per liter of i-fog, and one for $2.5 \times 10^{-3} \mathrm{~mol} / \mathrm{L}$.

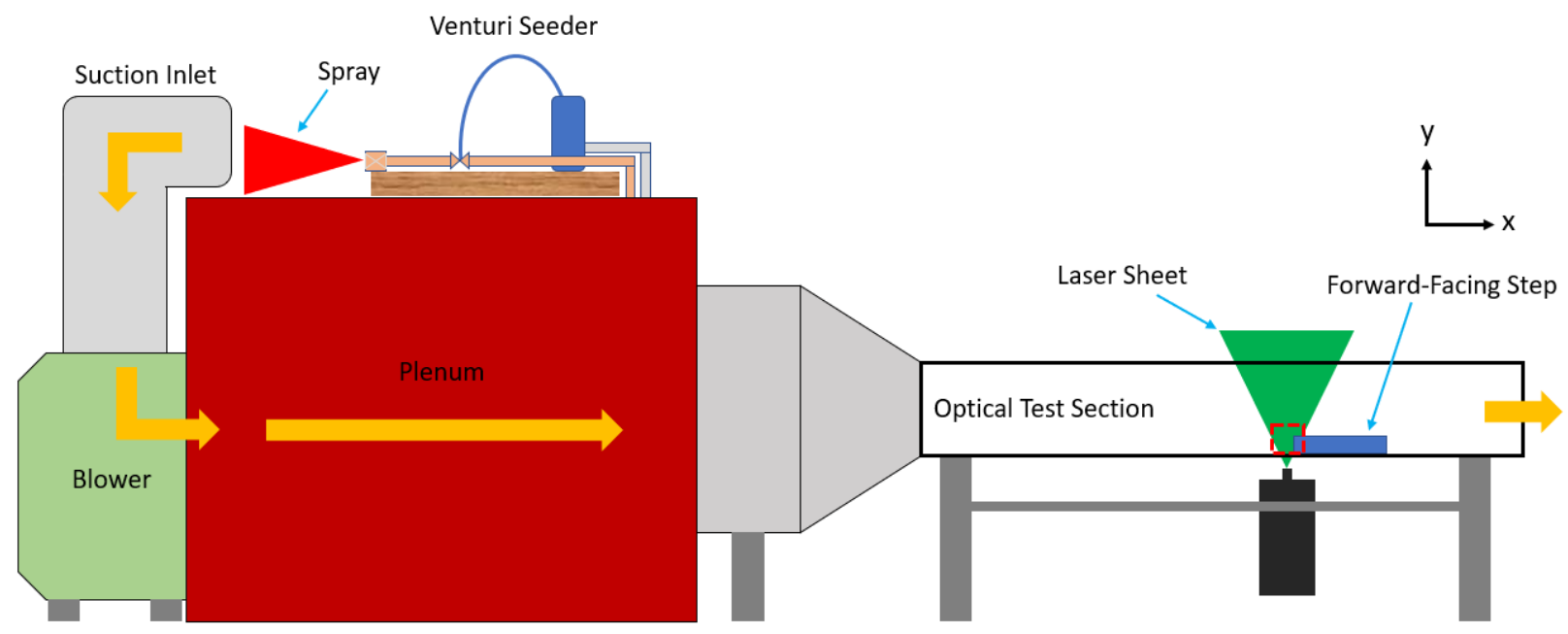

Figure 2: SBL wind tunnel PIV experiment setup (not to scale); red dashed square indicates camera FOV

PIV processing was done in LaVision's DaVis 8.4 software. The time domain filter method developed by Sciacchitano and Scarano (2014) was used on all data sets to improve performance in flareaffected regions. Acquired images were processed with a multi-pass technique; the first pass used 64 x 64 pixel interrogation windows with 50\% overlap, and the second pass used $32 \times 32$ pixel interrogation windows with $87 \%$ overlap. Spurious vectors were detected with the peak ratio $Q$, or the ratio of the highest to next-highest displacement correlation peak for a given interrogation window. Vectors were considered "spurious" if they had a $Q$ of 1.3 or greater. Additional spurious vector detection was performed with the median test introduced by Westerweel (1994). Vectors were removed if their residual value, $r$, was greater than 2 . Removed vectors were replaced with interpolated data.

\section{Results}

As previously stated, the goals of the experiments were to prove the suitability of the generated particles for PIV, and to demonstrate promising fluorescent PIV performance in regions close to the acrylic lower wall of the wind tunnel which are normally affected by surface flare. In this section, the 
former is addressed with statistical measurements from the TSI 3321 APS, and the latter is shown through raw PIV images along with comparisons of PIV-based boundary layer profiles and skin friction coefficients for fluorescent and Mie PIV cases.

\subsection{Particle Sizes}

The TSI 3321 APS collected 721,602 particles; statistical size parameters of the particles are displayed in Table 2, and a histogram of aerodynamic diameters is presented in Figure 3. Note that the APS was unable to determine diameters below $0.523 \mu \mathrm{m}$, which included nearly $8 \%$ of particles by number concentration; this created a possible bias in statistical results. Despite this, the aerodynamic median diameter was found to be $0.725 \mu \mathrm{m}$, and $92 \%$ of particles by number concentration were at, or below, $1 \mu \mathrm{m}$ in diameter. While ideal particle diameter limitations in any flowfield depend on the expected accelerations, Melling (1997) states that $1 \mu \mathrm{m}$ or less is typically suitable for gas flows. Further information on the performance of these particles can be obtained from relevant Stokes numbers. Using the aerodynamic median diameter of $0.725 \mu \mathrm{m}$, the particle lag timescale was found to be $1.7 \mu \mathrm{s}$. In the boundary layer flow used for this study's PIV experiment, this corresponds to a Stokes number of 0.0012 for the integral timescale and 0.3121 for the Kolmogorov timescale. Raffel et al. (2018) report that tracer particles with Stokes numbers below 0.1 typically yield acceptable accuracy, implying that the analyzed particles are suitable for measuring all but the smallest fluctuations in such a boundary layer. The APS data therefore suggests that the Venturi device's generated liquid particles are generally acceptable for PIV purposes for subsonic aerodynamics experiments at the stated pressure and needle valve settings.

Table 2: Statistical data for diameters of particles collected by TSI Aerodynamic Particle Sizer

\begin{tabular}{c|c} 
Statistical Parameter & Value $[\boldsymbol{\mu m}]$ \\
\hline Aerodynamic Median & 0.725 \\
Aerodynamic Mean & 0.933 \\
Geometric Mean & 0.829 \\
Geometric Standard Deviation & 1.540
\end{tabular}

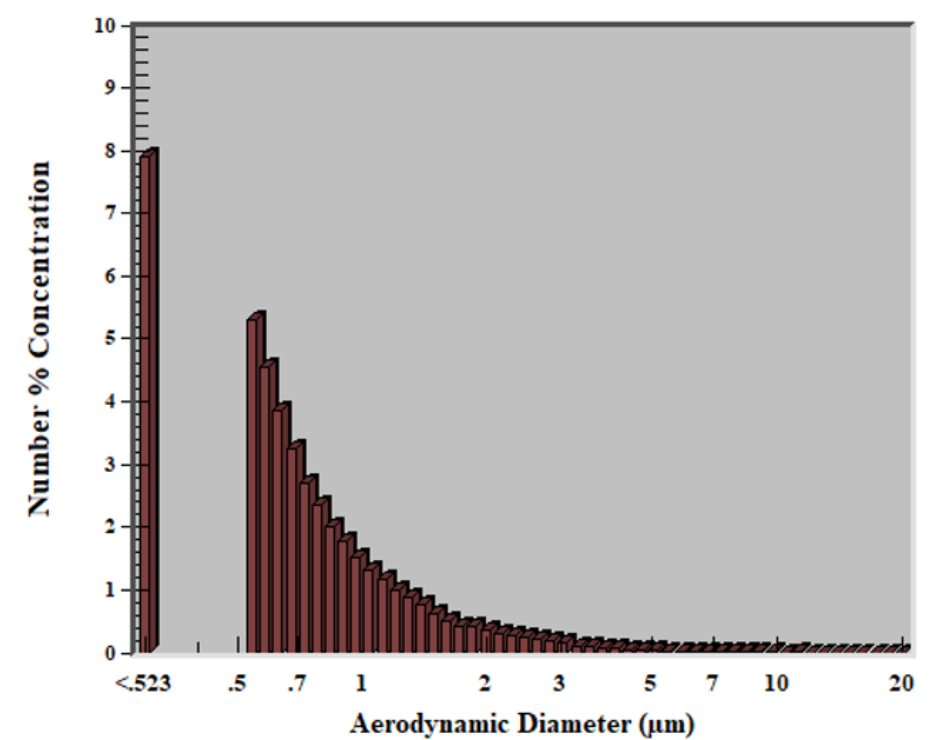

Figure 3: Histogram of aerodynamic diameters of particles collected by TSI 3321 APS

\subsection{PIV Imaging}

The PIV experiment was conducted to confirm the effectiveness of the fluorescent particles for PIV and to highlight differences between fluorescent and Mie PIV. Example instantaneous raw images 
for the four data sets are shown in Figure 4. In these images, both axes are nondimensionalized using the height $h$ of the aluminum step. The lower tunnel wall, at $\mathrm{Y} / \mathrm{h}=0$, is highlighted with an artificial blue line. Notably, the liquid seeding particles for the $1.0 \times 10^{-2} \mathrm{~mol} / \mathrm{L}$ case were clearly visible even through the fluorescent filter which blocked the incident laser wavelength, as shown in Figure 4d. Particles for the $2.5 \times 10^{-3} \mathrm{~mol} / \mathrm{L}$ case were also visible (Figure 4c), though dimmer. The fluorescent PIV images, as expected, had lower general particle intensities than their Mie PIV counterparts - most $1.0 \times 10^{-2} \mathrm{~mol} / \mathrm{L}$ fluorescent particles were roughly 50 times less intense than Mie PIV particles at the same aperture setting (f/2.8). They also appeared to show fewer particles, but this was an artifact of the images' unchanged intensity axes. Some small near-wall regions of brightness were visible in the fluorescent PIV images; these were likely accumulations of out-of-focus fluorescent particles on the wall itself, as they were seen to grow and shift over time.

As expected, background intensity counts were considerably higher for the Mie f/2.8 case than the other three. Figure 4a shows significant flare in the near-wall region, despite the transparent acrylic. This was exacerbated at lower $\mathrm{X} / \mathrm{h}$ values, closer to the reflective forward-facing step, which itself was located at $\mathrm{X} / \mathrm{h}=0$. Figure $4 \mathrm{~b}$ shows that reducing the aperture by 6 full stops to $\mathrm{f} / 22$ (thus reducing passing light by a factor of 64) significantly mitigated the flare, but horizontal flare streaks and a discernable thin layer of extreme brightness just above $\mathrm{Y} / \mathrm{h}=0$ were still visible. In Figures $4 \mathrm{c}$ and $4 \mathrm{~d}$, the fluorescent cases, many of the near-wall flare issues were reduced or eliminated. The region of extreme brightness just above $\mathrm{Y} / \mathrm{h}=0$ appeared thinner, and the horizontal streaks of flare visible in Figure $4 \mathrm{~b}$ were no longer present.

(a) Mie, $f / 2.8$

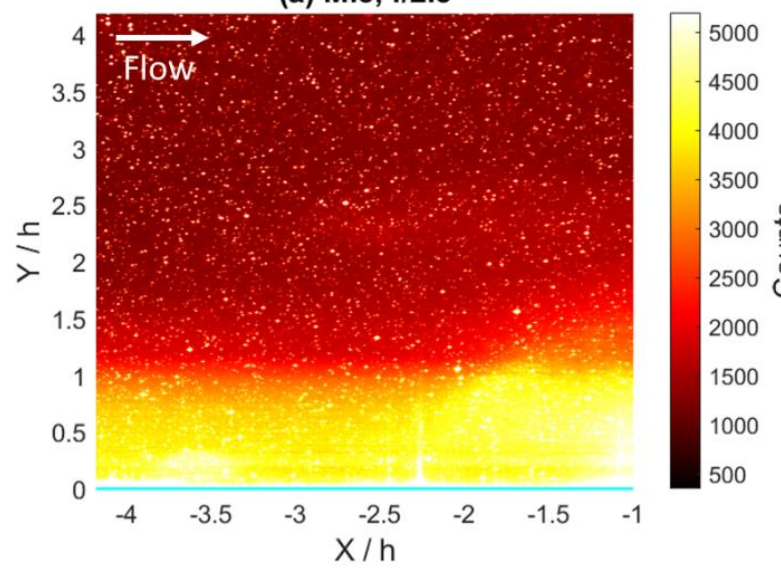

(c) Fluorescent $\left(2.5 \times 10^{-3} \mathrm{~mol} / \mathrm{L}\right), \mathrm{f} / 2.8$

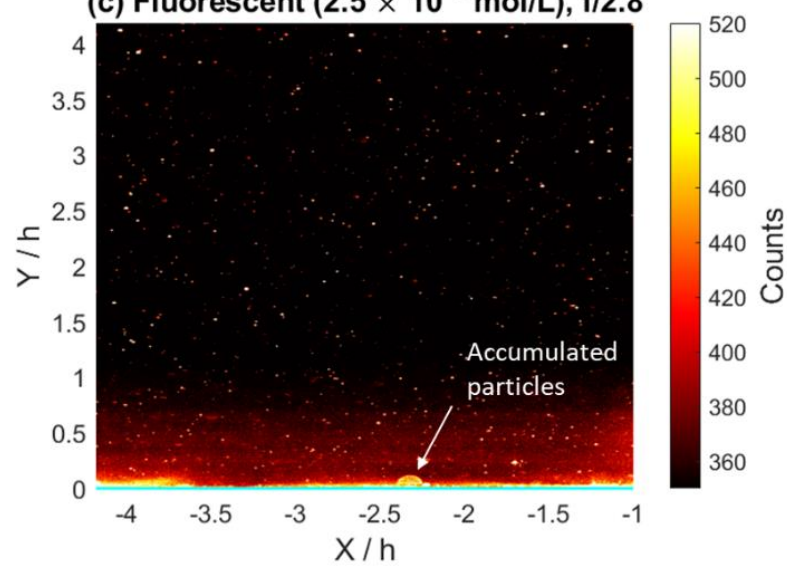

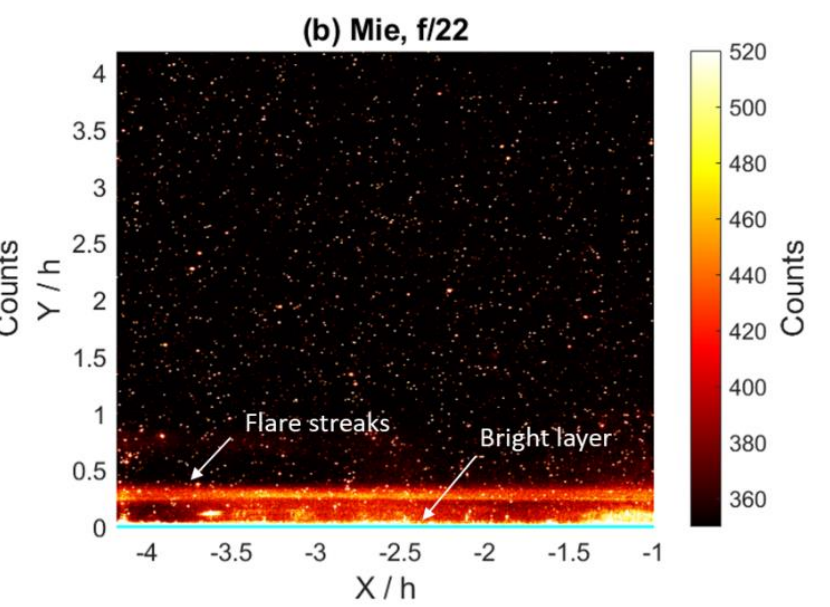

(d) Fluorescent $\left(1.0 \times 10^{-2} \mathrm{~mol} / \mathrm{L}\right), \mathrm{f} / 2.8$

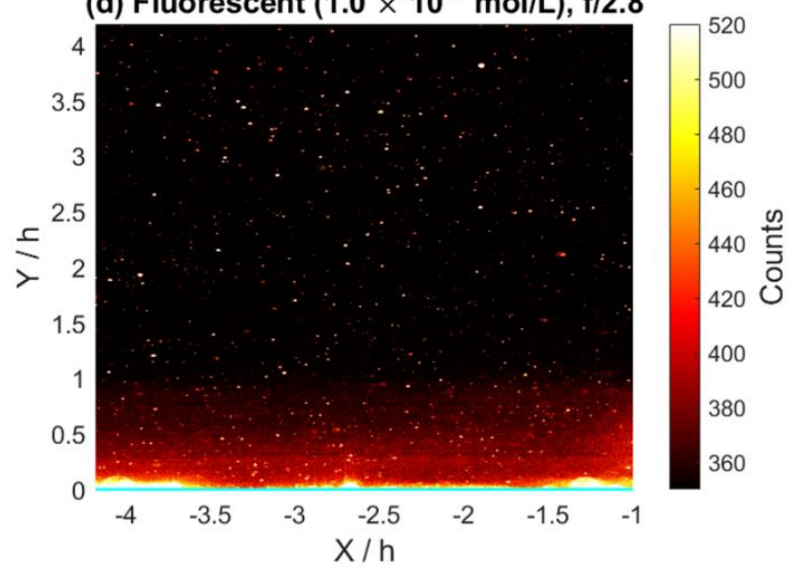

Figure 4: Example raw PIV images for select region upstream of forward-facing step $(\mathrm{X} / \mathrm{h}=0)$; tunnel wall $(\mathrm{Y} / \mathrm{h}=0)$ highlighted with blue line 


\subsection{Boundary Layer Comparisons}

Boundary layer data were extracted for the streamwise mean velocities of each data set. Figure 5 is a plot of the boundary layer profiles at an X-position that was 5.5 step heights upstream of the step (X/h $=-5.5$ ), where step-induced pressure gradient effects would be the least amongst the analyzed stations. The plot is presented in common wall coordinates, which normalize the velocity and height using friction velocity, which itself relates to the wall shear stress and velocity gradient as $\tau_{w}=\left.v \frac{\partial U}{\partial Y}\right|_{y=0}=\rho u_{\tau}^{2}$. The friction velocities were determined using the well-known Clauser chart method [Clauser (1956)], which assumes that the velocity profiles follow a universal logarithmic form in the "log-law" region, chosen here to be $35<\mathrm{y}^{+}<100$. For comparison throughout the near wall region, the Spalding profile [Spalding (1961)] was also plotted; this profile blends the viscous sublayer (where $\mathrm{u}^{+}=\mathrm{y}^{+}$) and the log-law region with a fit through the buffer region between them.

Figure 5 shows good agreement between the Spalding profile, Mie f/22 PIV, and fluorescent f/2.8 $\mathrm{PIV}$ at $1.0 \times 10^{-2} \mathrm{~mol} / \mathrm{L}$. Below the log-law region, these profiles nearly match the Spalding profile as it transitions towards the viscous sublayer. This is not the case for Mie f/2.8 PIV and fluorescent f/2.8 PIV at $2.5 \times 10^{-3} \mathrm{~mol} / \mathrm{L}$, which both diverge from the Spalding profile in the buffer region. The clear improvement between Mie f/2.8 PIV and Mie f/22 PIV suggests that the effects of increased laser flare significantly hampered f/2.8 PIV measurements near the wall. There exists a similar clear improvement between the fluorescent PIV at $2.5 \times 10^{-3} \mathrm{~mol} / \mathrm{L}$ and $1.0 \times 10^{-2} \mathrm{~mol} / \mathrm{L}$; the increased dye concentration is necessary for accurate PIV results. The fluorescent yield of tracer particles is generally proportional to dye concentration [Lemoine et al. (1999)]; so, there must exist a threshold below which PIV performance is negatively affected. However, dye concentrations in the present study were far higher than those used by Lemoine et al., a regime where significant self-quenching and loss of fluorescence yield would normally be expected. It is possible that the tracer particles experienced low levels of excitation flux, only absorbing a small amount of the laser energy and thus keeping the particles in the regime where fluorescent yield remains proportional to dye concentration.

The forward-facing step created an adverse pressure gradient in the upstream near-wall region; the log-law region of the boundary layer profile could not necessarily be expected to match the Spalding profile closer to the step than $\mathrm{X} / \mathrm{h}=-5.5$. As shown by Aubertine and Eaton (2005) and Wang et al. (2016), boundary layer profiles are significantly affected by adverse pressure gradients at their higher $\mathrm{y}^{+}$ values. The general trend is for $\mathrm{u}^{+}$values in these regions to increase across the gradient. However, at lower $\mathrm{y}^{+}$values, the boundary layer profiles remain relatively unchanged across the gradient and continue to match the Spalding profile well. Thus, in the present study, the lowest values of the boundary layer profiles $\left(\mathrm{y}^{+}<11\right)$ were matched with the Spalding profile by way of adjusting the friction velocity at each axial station. The resulting profiles in wall scaling are shown in Figure 6 for all four PIV cases, in the same style as Wang et al. (2016). As expected, $\mathrm{u}^{+}$values in the upper regions tended to increase across the adverse pressure gradient (approaching $\mathrm{X} / \mathrm{h}=0$ ) due to the wall friction reduction.

A friction velocity was found at each axial station of each PIV case through the aforementioned adjustments. These were converted to skin friction coefficients, as $c_{f}=2 u_{\tau}{ }^{2} / u_{\text {ref }}^{2}$, where $u_{\tau}$ is the friction velocity and $u_{\text {ref }}$ is a reference velocity, chosen here to be the freestream velocity of $27 \mathrm{~m} / \mathrm{s}$. The resulting friction coefficients are plotted in Figure 7. All PIV cases demonstrated a general decreasing trend as the step was approached, consistent with previous computational and oil-film interferometry findings regarding skin friction coefficient in an adverse pressure gradient [Na and Moin (1998), Pailhas et al. (2009)]. The data for Mie f/2.8 PIV and fluorescent PIV at $2.5 \times 10^{-3} \mathrm{~mol} / \mathrm{L}$ descend erratically, however; this conflicts with the smoothness and consistency of the curves found by Na and Moin and Pailhas et al. The data for Mie f/22 PIV has less variance as the flow approaches the step, but the friction coefficient of the fluorescent PIV at $1.0 \times 10^{-2} \mathrm{~mol} / \mathrm{L}$ exhibits the most physically consistent variation across the pressure gradient. 


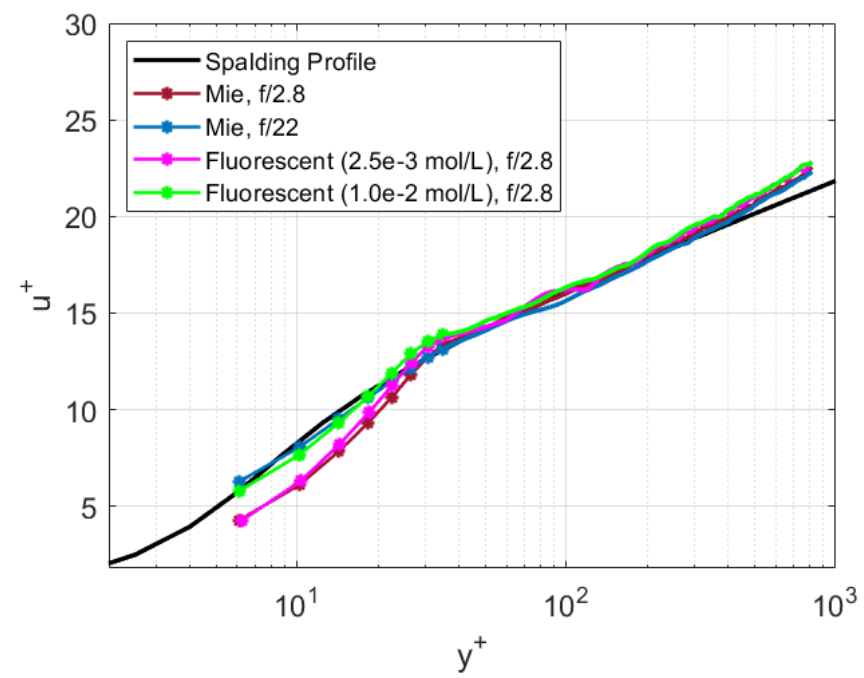

Figure 5: Boundary layer profiles for all four cases far upstream of step (symbols not shown above $\mathrm{y}^{+}=$ 13); Spalding profile shown for comparison

(a) Mie, f/2.8

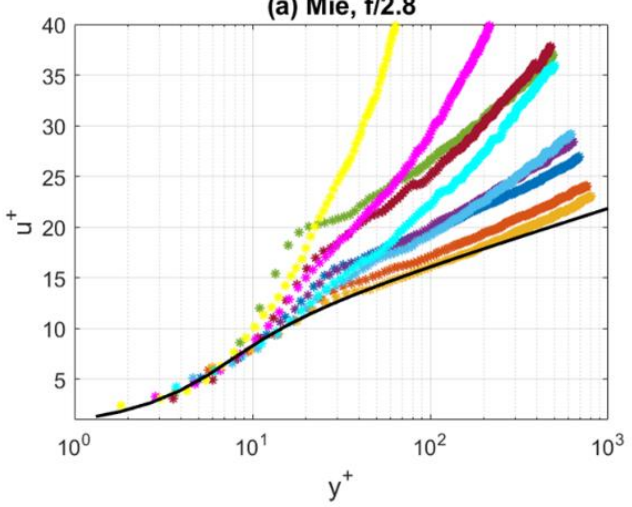

(c) Fluorescent $\left(2.5 \times 10^{-3} \mathrm{~mol} / \mathrm{L}\right), \mathrm{f} / 2.8$

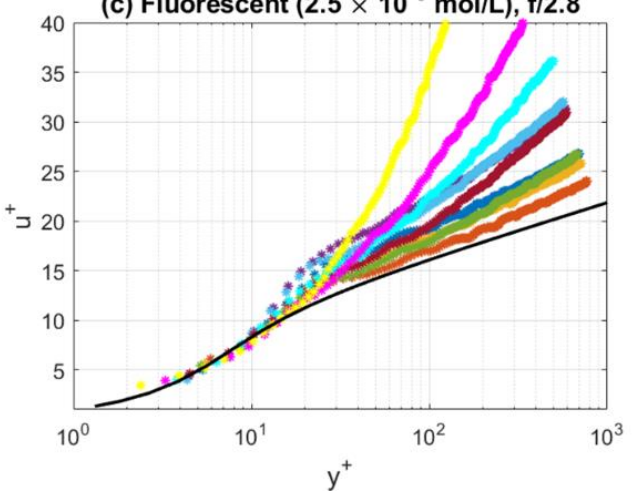

(b) Mie, f/22

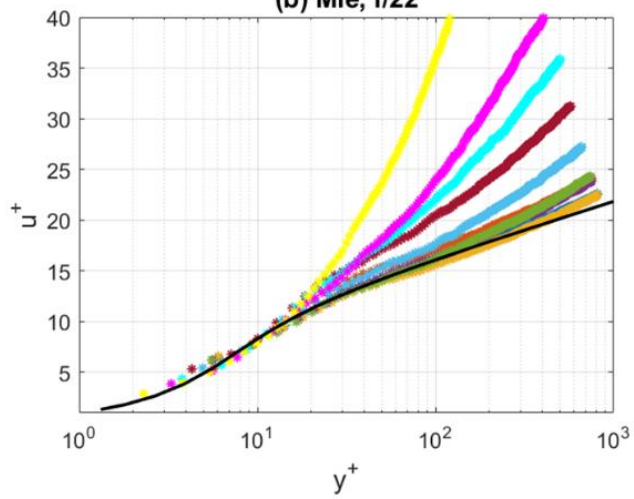

(d) Fluorescent $\left(1.0 \times 10^{-2} \mathrm{~mol} / \mathrm{L}\right), \mathrm{f} / 2.8$

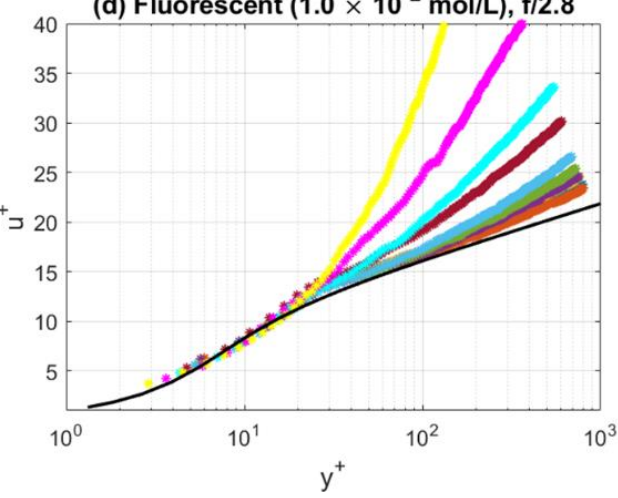

* $\quad \mathrm{X} / \mathrm{h}=-5.5$

* $\quad \mathrm{X} / \mathrm{h}=-5.0$

* $\mathrm{X} / \mathrm{h}=-4.5$

* $\quad \mathrm{X} / \mathrm{h}=-4.0$

* $\quad \mathrm{X} / \mathrm{h}=-3.5$

* $\mathrm{X} / \mathrm{h}=-3.0$

* $\mathrm{X} / \mathrm{h}=-2.5$

* $X / \mathrm{h}=-2.0$

* $\mathrm{X} / \mathrm{h}=-1.5$ $\mathrm{X} / \mathrm{h}=-1.0$

Spalding Profile

Figure 6: Boundary layer development across the adverse pressure gradient approaching the step; Spalding profile shown for comparison 


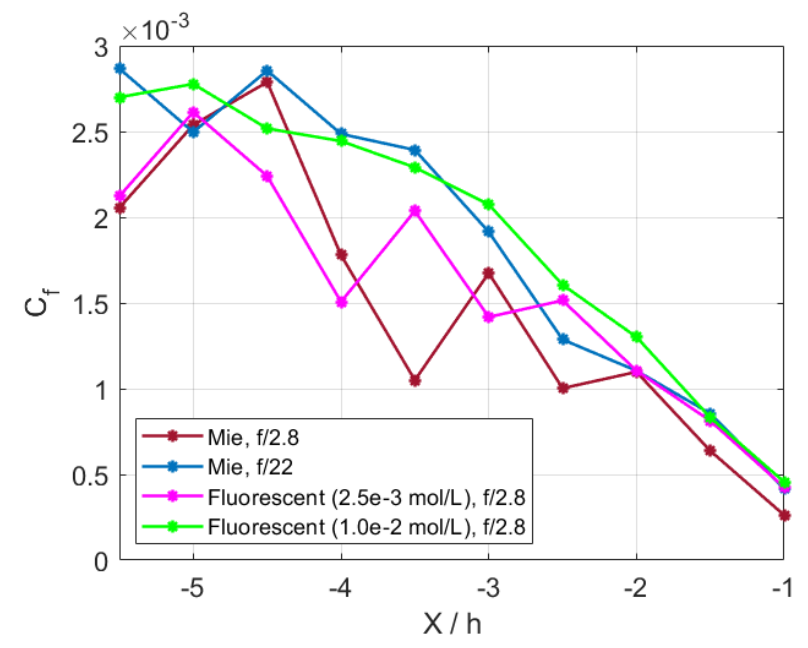

Figure 7: Skin friction coefficients for all PIV cases upstream of the step

\section{Conclusions}

Aerosolized fluorescent particles generated using a Venturi-type atomizer were found to be suitable for PIV purposes; this was demonstrated in multiple ways. First, the particles were found to be of acceptable size for PIV in many aerodynamic flows, with a median aerodynamic diameter of $0.725 \mu \mathrm{m}$. Second, the fluorescent particles with a dye concentration of $1.0 \times 10^{-2} \mathrm{~mol} / \mathrm{L}$ produced raw PIV images with good contrast when a filter was used to eliminate the incident $532 \mathrm{~nm}$ laser light and long-pass Stokes-shifted fluorescence from the particles. Using the fluorescence-passing filter resulted in significantly reduced laser flare in near-wall regions. Third, the measured boundary layer streamwise mean velocity profile of the fluorescent PIV case with a dye concentration of $1.0 \times 10^{-2} \mathrm{~mol} / \mathrm{L}$ was in close agreement with the theoretical Spalding profile.

This method of liquid fluorescent PIV shows promise for potentially generating more accurate results in near-wall regions of a flow. The streamwise evolution of skin friction obtained from fits to the Spalding profile for $y^{+}<11$ showed noticeable reduced variance in the $1.0 \times 10^{-2} \mathrm{~mol} / \mathrm{L}$ case throughout the adverse pressure gradient compared with the three other cases considered. The smooth and monotonic decrease of the skin friction coefficients from the $1.0 \times 10^{-2} \mathrm{~mol} / \mathrm{L}$ fluorescent PIV case was in qualitative agreement with trends observed in previous studies of adverse pressure gradients using computational means and oil-film interferometry.

Taken together, the results indicate that using a dye concentration of $1.0 \times 10^{-2} \mathrm{~mol} / \mathrm{L}$ and the Venturi-based atomizer is a viable, scalable, and cost-effective approach for implementing fluorescencebased PIV for measurements near surfaces in aerodynamic flows. Potential future studies may investigate the method's use for PIV in complex geometries where flare is otherwise difficult to mitigate.

\section{References}

Acharya AS, Lowe KT, Ng WF, and Danehy PM (2021) Seeding Mechanism for High-Pressure Nozzles. In AIAA SciTech 2021 Forum - Virtual Event, January 11-21

Aubertine CD and Eaton JK (2005) Turbulence Development in a Non-Equilibrium Turbulent Boundary Layer with Mild Adverse Pressure Gradient. Journal of Fluid Mechanics 532:345-364

Cadel DR, Shin D, and Lowe KT (2016) A Hybrid Technique for Laser Flare Reduction. In $54^{\text {th }}$ AIAA Aerospace Sciences Meeting - San Diego, CA, USA, January 4-8

Chennaoui M, Angarita-James D, Ormsby MP, Angarita-James N, McGhee E, Towers CE, Jones AC, and Towers DP (2008) Optimization and Evaluation of Fluorescent Tracers for Flare Removal in Gas-Phase Particle Image Velocimetry. Measurement Science and Technology 19:115403 
Clauser F (1956) The Turbulent Boundary Layer. Advances in Applied Mechanics 4:1-51

Lemoine F, Antoine Y, Wolff M, and Lebouche M (1999) Simultaneous Temperature and 2D Velocity Measurements in a Turbulent Heated Jet using Combined Laser-Induced Fluorescence and LDA. Experiments in Fluids 26:315-323

Maisto PMF, Lowe KT, Byun G, Simpson R, Verkamp M, Danley JE, Koh B, Tiemsin PI, Danehy PM, and Wohl CJ (2013) Characterization of Fluorescent Polystyrene Microspheres for Advanced Flow Diagnostics. In $43^{\text {rd }}$ Fluid Dynamics Conference - San Diego, CA, USA, June 24-27

Melling A (1997) Tracer Particles and Seeding for Particle Image Velocimetry. Measurement Science and Technology 8:1406-1416

Na Y and Moin P (1998) The Structure of Wall-Pressure Fluctuations in Turbulent Boundary Layers with Adverse Pressure Gradient and Separation. Journal of Fluid Mechanics 377:347-373

Pailhas G, Barricau P, Touvet Y, and Perret L (2009) Friction Measurement in Zero and Adverse Pressure Gradient Boundary Layer using Oil Droplet Interferometric Method. Experiments in Fluids 47:195-207

Paterna E, Moonen P, Dorer V, and Carmeliet J (2013) Mitigation of Surface Reflection in PIV Measurements. Measurement Science and Technology 24:057003

Pedocchi F, Martin JE, and Garcia MH (2008) Inexpensive Fluorescent Particles for Large-Scale Experiments using Particle Image Velocimetry. Experiments in Fluids 45:183-186

Petrosky BJ, Lowe KT, Bardet PM, Tiemsin PI, Wohl CJ, Danehy PM, and Andre M (2015) Particle Image Velocimetry Applications using Fluorescent Dye-Doped Particles. In $53^{\text {rd }}$ AIAA Aerospace Sciences Meeting - Kissimmee, FL, USA, January 5-9

Poussou S and Plesniak MW (2006) Near-Field Flow Measurements of a Cavitating Jet Emanating from a Crown-Shaped Nozzle. Journal of Fluids Engineering 129:605-612

Raffel M, Willert CE, Scarano F, Kahler CJ, Wereley ST, and Kompenhans J (2018) Particle Image Velocimetry: A Practical Guide. Springer

Sciacchitano A and Scarano F (2014) Elimination of PIV Light Reflections via a Temporal High-Pass Filter. Measurement Science and Technology 25:084009

Spalding D (1961) A Single Formula for the Law of the Wall. Journal of Applied Mechanics 28:455-458

Uzol O, Chow YC, Katz J, and Meneveau C (2002) Unobstructed Particle Image Velocimetry

Measurements within an Axial Turbo-Pump using Liquid and Blades with Matched Refractive Indices. Experiments in Fluids 33:909-919

Wang QC, Wang ZG, and Zhao YX (2016) On the Impact of Adverse Pressure Gradient on the Supersonic Turbulent Boundary Layer. Physics of Fluids 28:116101

Wernet MP (2000) Development of Digital Particle Imaging Velocimetry for Use in Turbomachinery. Experiments in Fluids 28:97-115

Westerweel J (1994) Efficient Detection of Spurious Vectors in Particle Image Velocimetry Data. Experiments in Fluids 16:236-247

Willert CE (2015) High-Speed Particle Image Velocimetry for the Efficient Measurement of Turbulence Statistics. Experiments in Fluids 56:17

Wohl CJ, Kiefer JM, Petrosky BJ, Tiemsin PI, Lowe KT, Maisto PMF, and Danehy PM (2015) Synthesis of Fluorophore-Doped Polystyrene Microspheres: Seed Material for Airflow Sensing. ACS Applied Materials \& Interfaces 7:20714-20725 